\title{
UNIFIED ALTERED FREE VOLUME STATE MODEL FOR TRANSPORT PHENOMENA IN POLYMERIC MEDIA
}

\author{
R.A. Mashelkar and M.G. Kulkarni \\ National Chemical Laboratory, Pune 411008 , India
}

\begin{abstract}
An Altered Free Volume State (AFVS) model has been proposed for analysing and correlating a variety of transport phenomena in polymeric media. The key concept is the calculation of the alteration of the free volume state of the parent medium with respect to a carefully defined reference state. This approach enables the prediction of the influence of alteration of many variables including the changes in the physico-chemical structural attributes of the polymeric systems on a surprisingly large variety of transport phenomena. The successful application of this unified model has been demonstrated by analysing exhaustive experimental data.
\end{abstract}

\section{INTRODUCTION}

A considerable effort has been spent in analysing and modelling transport processes involving viscous flow, diffusional transport, thermal conduction, el ectrical conduction etc. in polymeric media. Apart from a significant number of experimental investigations which exists, a large number of predictive or correlative equations, which are based on either molecular or phenomenological considerations have been proposed. In most such cases, the theoretical developments or modelling efforts pertain to a specific transport property or a specific type of polymeric medium. It appears that although the physical forms of the diverse media in which polymers can exist can range from polymer solutions at one end to solid polymers at the other, there is a strong case to build up a unified framework for correlating transport properties. We examine such a possibility here.

The central concept used in our work can be elaborated as follows. We assume that the free volume state of the medium is the key factor in determining various transport properties. We then assume that a given parent matrix has a particular state of free volume which can be altered by making either physical or chemical modifications or by altering the state variables such as temperature, pressure etc. In the case of solid polymers such modifications could be done by introducing crystallites [ semicrystalline polymers], crosslinking [network polymers], filler addition [filled polymers] etc. We consider that the free volume state of the parent matrix has been modified by such an alteration or in other words we have a medium with an 'altered free volume state'. We assume that the transport properties of the medium under consideration are the same as that of the parent matrix in the altered state. Since the free volume has been chosen to characterize the altered state, the model we build up can be termed as an 'altered free volume state model' [AFVS model].

The physical basis for the calculation of such an alteration in the free volume state forms the key concept in correlating diverse transport properties. In Table 1 we present a matrix of some of the specific variables which affect the properties of the matter in different states. Development of a unique framework which enables correlation or prediction of all these variables on the transport properties listed out is indeed difficult. However, even the limited success that we have been able to achieve in this regard appears to suggest a great potential for future analysis.

We wish to emphasize here that free volume models for transport phenomena in polymeric media have been indeed developed in the past. However, we believe that the range of systems considered in this work and the method of estimation of the altered free volume has not been used in the past. Although in most cases our model will enable only a relative evaluation of the influence of system variables, in some cases we have been able to obtain semi-predictive relationship with only a single adjustable (but physically meaningful) parameter. The main drawback of a unified framework might appear to be the fact that since a broad canvass is being covered, the intricate details of an individual transport process might be somewhat lost. 
TABLE 1. Influence of structural variables on transport properties*

\begin{tabular}{|c|c|c|c|}
\hline & Viscosity & Diffusivity & Thermal conductivity \\
\hline 1. Molecular weight & $\leftarrow$ & 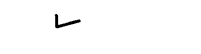 & レ \\
\hline $\begin{array}{l}\text { 2. Molecular weight } \\
\text { distribution }\end{array}$ & レ & $x$ & $x$ \\
\hline 3. Branching & $\leftarrow$ & $x$ & $x$ \\
\hline 4. Crosslinking & $x$ & ᄂ & $レ$ \\
\hline 5. Crystallinity & NA & レ & $\leftarrow$ \\
\hline 6. Orientation & NA & レ & $\leftarrow$ \\
\hline $\begin{array}{l}\text { 7. Polymer concentration } \\
\text { in blends or in } \\
\text { solution }\end{array}$ & レ & ᄂ & レ \\
\hline 8. Solute shape and size & NA & L & NA \\
\hline
\end{tabular}

\section{VISCOSITY OF POLYMERIC SYSTEMS}

Innumerable theoretical developments and semi-empirical correlations have been proposed in the literature for correlating the viscous behaviour of fluids. With specific reference to polymers a large number of exhaustive reviews covering dilute polymer solutions [Ref.1], concentrated polymer solutions [Ref.2], polymer melts [Ref.3], colloidal suspensions [Ref.4], polymer latices [Ref.5] have appeared in the past.

The free volume model has been widely used in the literature to correlate the temperature effects [Ref. 6] as well as the composition dependence of viscosity [Ref. 7]. Fujita [7] adopted the free volume approach to correlate the composition dependence of diffusivity as well. The addition of a diluent to the polymer was assumed to increase the free volume of the polymer-diluent system, which in turn led to an increase in the diffusivity and decrease in viscosity. The model has been successful in correlating diffusivity and viscosity behaviour of highly concentrated solutions also. Recently, Chitrangad and Osmers [8] showed that a similar approach can be adopted for dilute polymer solutions choosing pure solvent as the frame of reference.

According to the free yolume model the viscosity of the medium could be related to the fractional free volume by the relationship :

$$
\eta_{0}=A \exp \frac{B \eta}{F_{0}}
$$

where $\eta_{0}$ denotes the viscosity of the medium having a fractional free volume $f_{0}$. A denotes the preexponential factor and $\mathrm{Bn}$ is related to the hole size required to accommodate a segment taking part in viscous flow. Assume that we add to this base medium a component constituting a volume fraction $\emptyset$. The component could be a rigid particle and the base medium could be a solvent or a polymer solution or a molten polymer. This addition will result in the alteration of the free volume of the base medium due to [1] the physical obstruction effect leading directly to loss of free volume [2] immobilization of the molecules of the medium in the immediate neighbourhood of the solid particle due to the non-slip condition. We may assume that the reduction in the free volume due to immobilization is proportional to the volume fraction of the added component. The altered

Note $X$ : denotes lack of data

NA : denotes not applicable 
free volume state for the new medium could be now defined as

$$
f=f_{0}(1-\emptyset)-\beta \varphi
$$

where $\beta$ is a proportionality constant reflecting on the loss of mobility due to the immobilization. The viscosity in this new altered free volume state would be now given by:

$$
\eta=A \exp \frac{B_{\eta}}{f}
$$

Combining equations [1], [2] and [3] we obtain :

$$
\ln \frac{\eta}{\eta_{0}}=\frac{\left[\frac{B_{\eta}}{f_{0}}\left(1+\frac{B_{\eta}}{f_{0}}\right)\right] \varphi}{1-\left(1+\frac{B_{\eta}}{f_{0}}\right) \emptyset}
$$

and reorganizing :

$$
\frac{1}{\ln \frac{\eta}{\eta_{0}}}=\frac{1}{\left[\frac{B_{\eta}}{f_{0}}\left(1+\frac{\beta}{f_{0}}\right)\right] g}-\frac{f_{0}}{B_{\eta}}
$$

equation [5] suggests that within the context of this altered free volume state model, a plot of $\left(1 n \pi / \eta_{0}\right)^{-1}$ vs $\emptyset^{-1}$ should be 1 inear. A special case of equation [5] arises when the effect due to immobilization becomes marginal. In this case $\beta$ is approximately zero and we obtain :

$$
\ln \frac{\eta}{\eta_{0}}=\frac{B_{\eta}}{f_{0}} \quad\left(\frac{\emptyset}{1-\emptyset}\right)
$$

Verification of AFVS model for suspensions

The validity of equation [5] (or [6]) has been tested by us for a large number of suspensions. Three classes of systems have been examined : suspensions in Newtonian media, in polymer solutions and in polymer melts. Some typically successful data are shown in Figs. 1 and 2 .

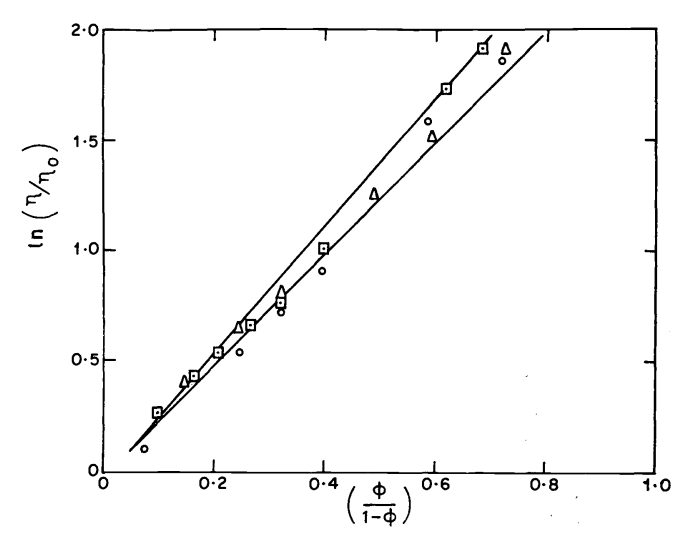

Fig. 1. Viscosity correlation for glass sphere suspensions in sucrose solution I (o), sucrose solution II $(\Delta)$ ) and white oil ( $\square$ ) (Data : Ref.9).

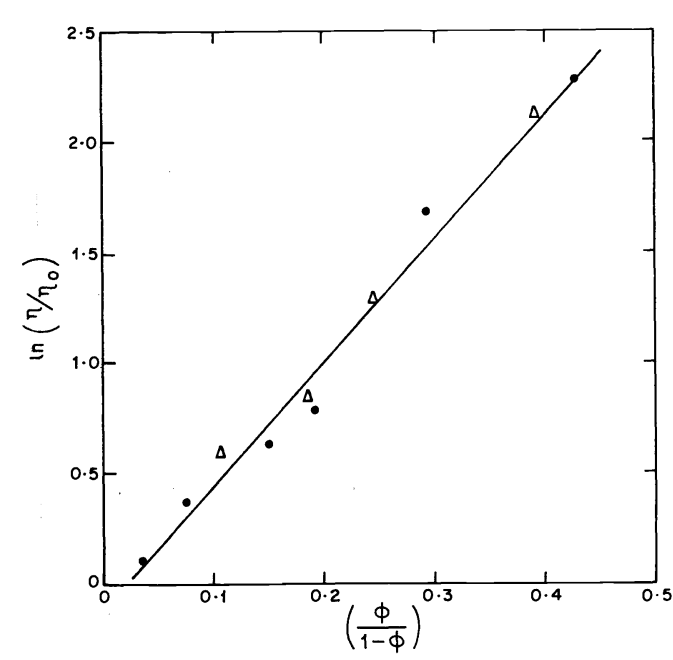

Fig.2. Viscosity correlation for calcium carbonate filled polyethylene $(\bullet)$ and polystyrene $(\Delta)$ melts (Data : Ref. 10). 
In Fig. 1 we show data on reduced viscosity for glass sphere suspensions in sucrose solutions and white oil. In Fig. 2 we show the plot for calcium carbonate filled polyethylene and polystyrene melts. It is seen that the trends predicted by equation [5] are verified.

\section{Verification of AFVS model for polymer blends}

There has been a considerable interest in the development of commercially yiable polymer blends and also their rheological characterization. A number of empirical correlations have been proposed in the literature to correlate this behaviour [Ref. 11]. We can use the AFVS model to develop an equation for prediction of composition dependence of viscosity in polymeric blends. It can be assumed again that the addition of a polymer to the parent polymer matrix causes an alteration in the free volume of the parent polymer matrix. Therefore, the viscosity behaviour of the polymer blends would be the same as that of the parent polymer matrix if evaluated at its altered free volume state. It can be readily deduced that if $\eta_{0}$ is the viscosity of the parent polymer to which is added a volume fraction $\emptyset_{\mathrm{BL}}$ of a second polymer then the resulting viscosity of the blend is given as

$$
\frac{1}{\ln \frac{\eta_{0}}{\eta_{B L}}}=\left[\frac{f(0)^{2}}{B_{\eta} \gamma}\right] \frac{1}{\theta_{B L}}+\frac{f(0)}{B_{\eta}}
$$

where $\emptyset_{\mathrm{BL}}$ denotes the volume fraction of the component added, $\mathrm{B} \eta$ denotes the jump factor, $f(0) \quad B L$ denotes the fractional free volume of the parent polymer and $\gamma$ denotes the difference in the fractional free volumes of the individual pure polymers being blended. Figures 3 and 4 show the plots which verify the validity of equation 7 . A feature common to these plots is that the blends comprise two components of the same type of polymer differing only in molecular weight. Hopefully, the same approach should work for compatible polymer blends also. This approach cannot be expected to work for two phase polymeric systems, in which one component exists as a discrete phase dispersed in a continuous phase. Such systems exhibit many complex phienomena including the presence of either a minimum or a maximum or both a minimum and a maximum depending upon the state of dispersion and morphology of the two phase polymer blends [Ref. 14].

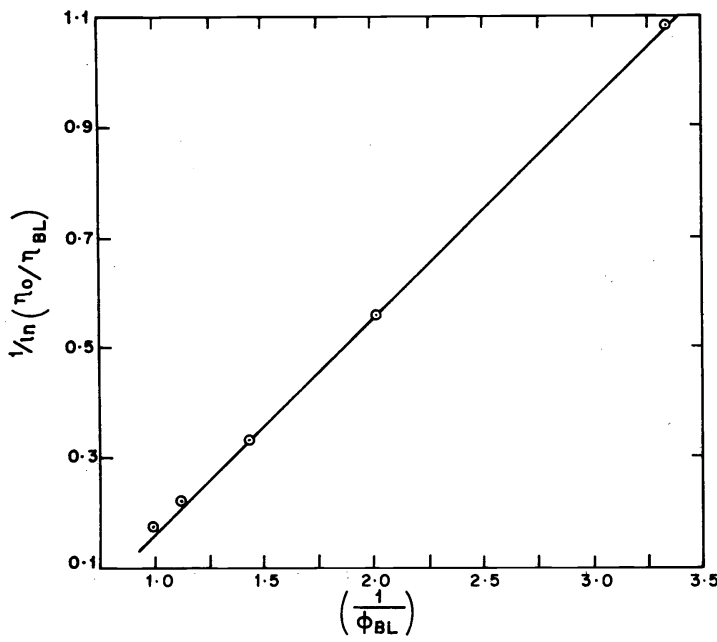

Fig. 3. Composition dependence of viscosity of polystyrene blends (Data : Ref. 12).

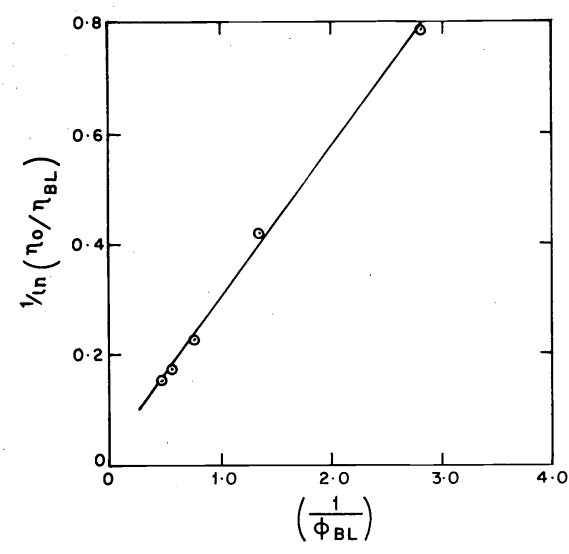

Fig. 4. Viscosity correlation for buty1 rubber blends (Data : Ref. 13).

Development and verification of AFVS model for polymer solutions

The data on viscous behaviour of polymer solutions could also be correlated in terms of an altered free volume approach. Equation [7] can be used for this purpose assuming that the addition of a polymer molecule essentially results in reduction of free volume of the parent matrix, which in this case would be the solvent. The parameter that needs to be defined carefully is the equivalent volume fraction of the polymer. Following Rudin and Strathdee [15] we can define it as :

$$
\emptyset_{p}=\frac{N_{A} V \text { solv }}{N E} \simeq \frac{C \epsilon}{\rho}
$$


where,

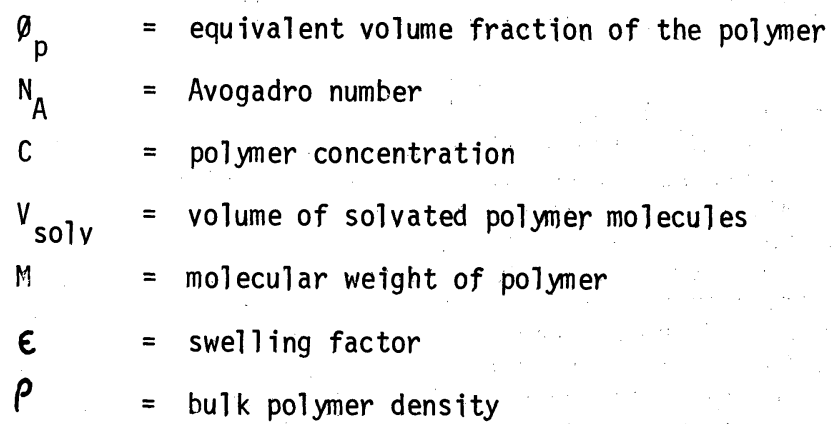

The swelling factor $€$ could be assumed to be a linear function of the volume fraction itself and will lie between two asymptotic 1 imits. At infinite dilution $\left[\emptyset_{0} \approx 0\right]$ the swelling factor has its maximum value $[\epsilon=\epsilon]$. When the occupied volume $\mathrm{p}^{\mathrm{p}}$ reaches that of a cubical packing for uniform spheres $\left[\emptyset_{p}=0.524\right]$ there is assumed to be no swelling
and $\epsilon=1$. We then obtain :

$$
\frac{1}{\epsilon}=\frac{1}{\epsilon_{0}}+\frac{C}{0.524 \rho} \frac{\epsilon_{0}-1}{\epsilon_{0}}
$$

$\epsilon_{0}$ can be calculated by using the Einstein equation

$$
\frac{\eta}{\eta_{0}}=1+2.5 \emptyset
$$

or equating $\emptyset=\emptyset_{p}$ and using the definition of intrinsic viscosity $[\eta]$, we get

$$
[\eta]=2.5 \frac{\emptyset_{\mathrm{p}}}{\mathrm{C}}=2.5 \frac{\epsilon_{0}}{\rho}
$$

We now use the AFVS model form as represented in equation [5] which can be recast into

$$
\frac{\eta}{\eta_{0}} \cdot \exp \frac{a \emptyset_{p}}{1-b \phi_{p}}
$$

with

$$
a=\frac{B_{\eta}}{f_{0}}\left(1+\frac{\beta}{f_{0}}\right) \quad b=1+\frac{\beta}{f_{0}}
$$

Expanding [13], we have

$$
\frac{\eta}{\eta_{0}}=1+\frac{a \theta_{p}}{1-b \theta_{p}}+\frac{\left(a \theta_{p}\right)^{2}}{2 !\left(1-b \theta_{p}\right)^{2}}
$$

Using the Einstein form in equation [14] for $\emptyset \ll 1$ and comparing with equation [10], we get $a=2.5$.

Equation [12] now takes the form

$$
\frac{\eta}{\eta_{0}}=\exp \frac{2.5 \theta_{p}}{1-b \theta_{p}}
$$

Note that equation [15] is the celebrated Mooney equation which has been extensively verified for suspensions. The parameter $b$ has been normally interpreted as the reciprocal of the maximum packing fraction. In terms of AFVS model we attach a new significance to $b$ as given in equation [13]. Using equations [12], [13] and [15] we deduce

$$
\frac{2.5}{\ln \frac{\eta}{\eta_{0}}}=\frac{1}{\emptyset_{p}}-b
$$

Within the context of the free volume model, equation [16] implies that a plot of 

$(\ln \eta / \eta)^{-1}$ vs $\left(\varphi_{p}\right)^{-1}$ should essentially be a linear one. Figure [5] shows the results of
analysiso for. naphthalene] in benzene at $30^{\circ} \mathrm{C}$.

Fig. 5. Viscosity correlation for poly (1-vinyl naphthalene) (๑) and poly (2-vinyl naphthalene) $(\Delta)$ in benzene at $30^{\circ} \mathrm{C}$. (Uata : Ref. 16).

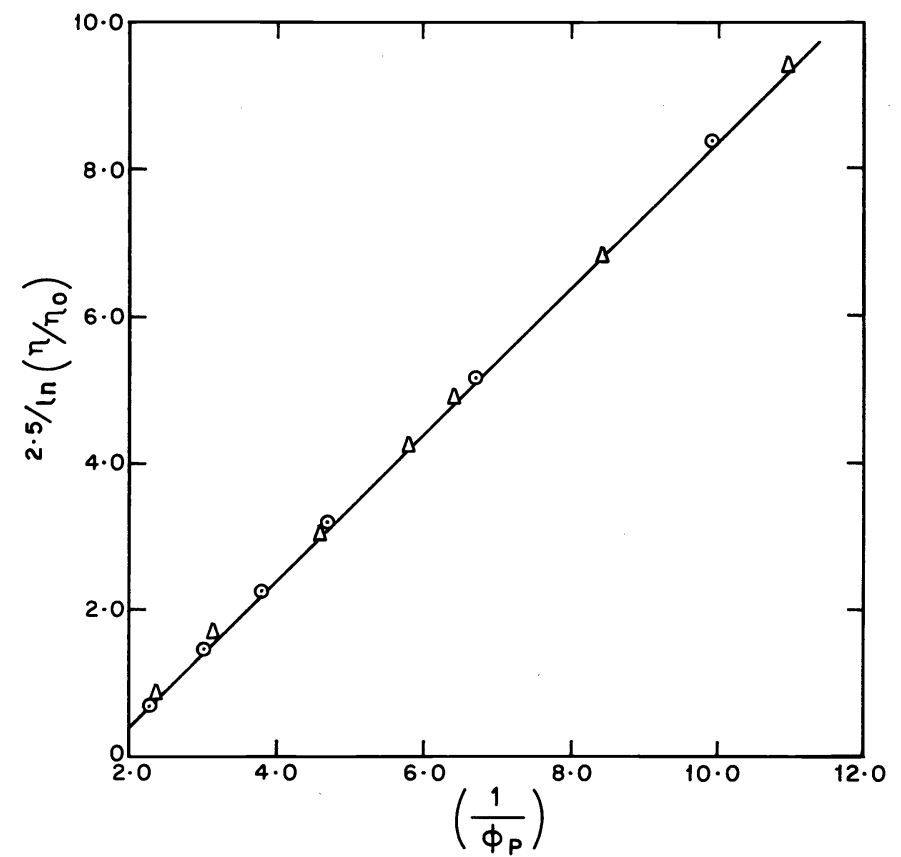

It is worth noting that the data for the two polymers get virtually superimposed. In fact we have observed that a large number of data in the literature could be correlated similarly [see Table 2]. In all the cases analysed by us the slopes of the curves appear to be very close to unity as is predicted by equation [16]. Note that similar range of values of jump factors has been reported by Fillers and Tschoegl [17].

\section{DIFFUSION IN POLYMERIC MEDIA}

The free volume approach has been used in the past to explain the concentration and temperature dependence of diffusivity in polymer-diluent systems [Ref. 6 and 7]. We show here that the AFVS model can be effectively used to model diffusion of a wide variety of solutes in diverse polymeric media.

In the first instance we shall consider the application to polymer solutions.

Diffusion of a small solute in dilute polymer solutions and gels

Concentration dependence of small solute diffusivity. Many investigations in the past have considered aspects of diffusion of a small solute in dilute polymer solutions. [see Astarita and Mashelkar (18)]. Invariably the addition of a polymer to the solvent results in reduction in diffusivity. We can consider that addition of a polymer results in alteration of the free volume state of the solvent and therefore diffusion occurs essentially in the solvent phase which has its free volume state altered. We might assume that the contribution of the polymer to the free volume is negligible. Then following considerations similar to the development of equation (6) we can deduce

$$
\ln \frac{D}{D_{0}}=-\frac{B_{d}}{f_{0}}\left[\frac{\varphi_{p}}{1-\varphi_{p}}\right]
$$

where $D$ is the diffusivity in the polymer solution, $D$ is the diffusivity in the solvent, $f_{0}$ is the fractional free volume of pure solvent, $B_{0} 0$ is the jump factor and $\theta_{p}$ is the polymer volume fraction in the solution. Sometimes ${ }^{d}$ we may have data on diffusivity at a 
TABLE 2. Results of analysis of polymer solutions data (Ref. 16)

\begin{tabular}{llllll}
\hline Polymer & $\begin{array}{l}\text { Concentration } \\
\text { range, g/dl }\end{array}$ & $\begin{array}{l}\text { Molecular } \\
\text { weight } \times 10^{-3}\end{array}$ & Slope & Intercept & $B_{\eta}$ \\
\hline PIVN-59 & $0.50-14.52$ & 155.3 & 0.9916 & -1.470 & 0.282 \\
P2VN-70 & $0.25-5.47$ & 563.4 & 0.9967 & -1.462 & 0.282 \\
P2VN-58 & $0.23-9.31$ & 306.1 & 0.9993 & -1.495 & 0.276 \\
P2VN-46 & $0.35-10.39$ & 182.5 & 0.9936 & -1.500 & 0.275 \\
P2VN-68 & $0.62-5.06$ & 64.0 & 0.9857 & -1.489 & 0.277 \\
P2VIN-61 & $0.64-14.50$ & 48.9 & 0.9800 & -1.416 & 0.291 \\
PVB-41 & $0.11-11.12$ & 106.5 & 0.9956 & -1.463 & 0.282 \\
PVB-71 & $0.29-12.14$ & 169.4 & 0.9984 & -1.413 & 0.292 \\
PVB-56 & $0.33-16.60$ & 103.0 & 1.0075 & -1.491 & 0.282 \\
PVB-51 & $0.87-20.93$ & 80.8 & 0.9962 & -1.464 & 0.282 \\
PVB-8036 & $1.96-24.90$ & 10.5 & 0.9747 & -1.582 & 0.262 \\
\hline
\end{tabular}

particular polymer concentration available, and we might wish to find the diffusivity at another concentration. This can be readily done if we again presume that the free volume state has been altered with reference to the original reference state. If $\phi^{*}$ is a reference concentration of the solvent then it can be readily shown that the relative diffusivities would be given by

$$
\frac{1}{\ln \frac{D}{D^{*}}}=\left[\frac{f\left(\varphi^{*}\right)^{2}}{B_{d} \Psi}\right] \quad \frac{1}{\emptyset-\varphi^{*}}+\frac{f\left(\varphi^{*}\right)}{B_{d}}
$$

where $\Psi$ represents the difference in the fractional free volumes of the reference medium (composition $\varphi^{*}$ ) and the pure solvent. Equations (17) or (18) can be used to correlate the diffusivity data.

Figure (6) shows a plot of diffusivity of turpentine in polyisobutylene - ethylbenzene system. It is seen that the trend predicted by equation (17) is borne out. Figure (7) shows the data on diffusion of chloropheniramine maleate in hydroxypropyl cellulose gels. It is seen that equation (18) is verified. A far more extensive range of data has been correlated by Kulkarni and ilashelkar (21) within the framework of equations (17) and (18).

Influence of polymer molecular weight on small solute diffusion. As emphasized earlier, the free volume model assumes that diffusion essentially occurs whenever a molecule finds a nole of large enough size in which it could be accommodated. This implies that the solute size could play an important role in the diffusion process depending upon the specific ratios of the length scales pertaining to the diffusant size and the hole size created by the cooperative movements of the polymer chains. It is rational to expect that for a long enough polymer chain any further increase in the chain length should not have any influence on the diffusion of small molecules. Figure 8 shows plot of $\left[\ln \left(D_{p} / D_{0}\right)\right]-1$ vs $1 / \emptyset-\emptyset^{*}$ for diffusion of 2,2 methylene-bis-[4 methyl-6 tert butyl phenol] $p_{\text {in }}$ polystyrene-ethylbenzene. A complete superposition of diffusivity data for polymers differing in molecular weights confirms our hypothesis. A similar plot for glycine-dextran gels also shows that $B_{d}$ for glycine is independent of the molecular weight of dextran (Ref. 23).

Influence of size and shape of the diffusant. We now discuss the significance of $B_{d}$. Since $B_{d}$ is the measure of the hole size required to accommodate the diffusing molecule, it might be expected to be a characteristic of the diffusing molecule. For a series of diffusants diffusing in a medium of fixed free volume state the diffusivity will be 


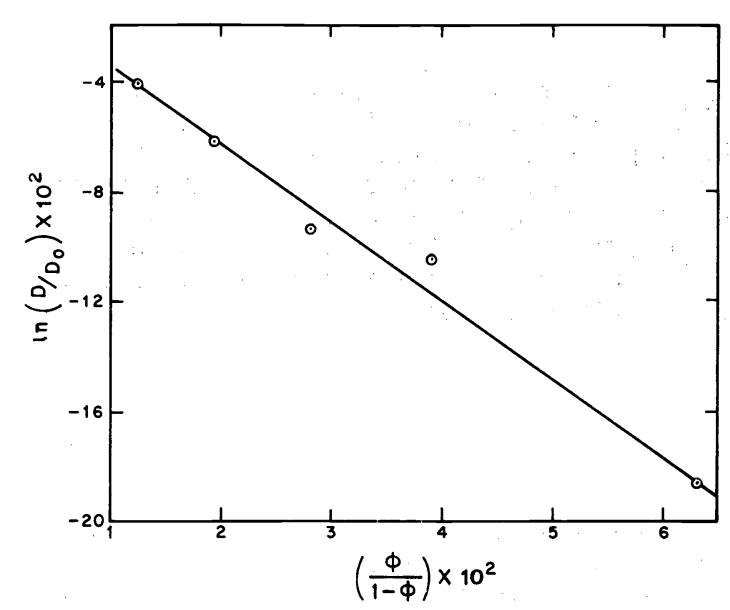

Fig. 6. Diffusivity correlation for turpentine in polyisobutylene-ethylbenzene system (Data : Ref. 19).

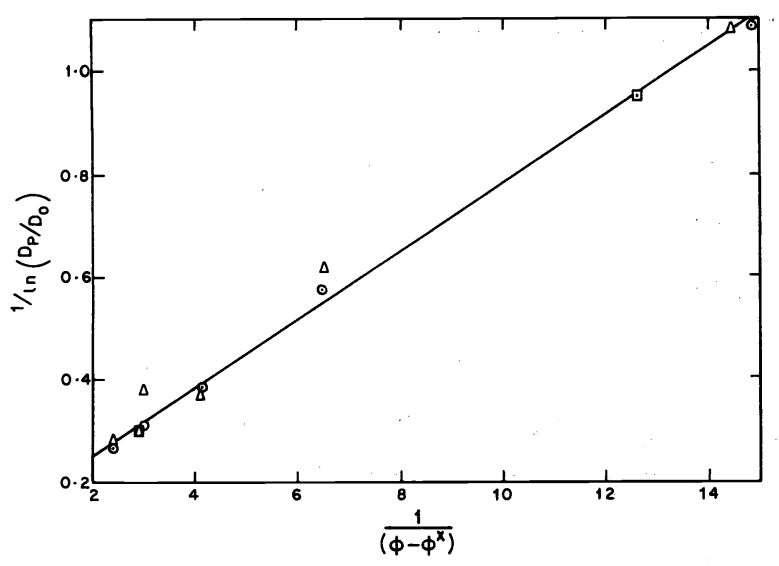

Fig. 8. Influence of molecular weight of polystyrene $-\left[5.5 \times 10^{4}(0), 1.08 \times 10^{6}\right.$ $(\triangle), 1.16 \times 10^{5}$ (口) $]$ on diffusivity of 2 , 2 methylene bis [ 4 methy 1 - 6 tert butyl pheno1]. $\emptyset^{*}=0.41$ (Data : Ref. 22).

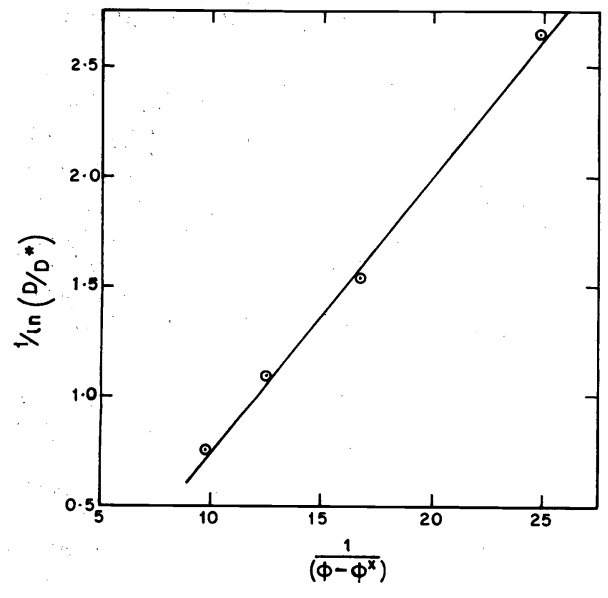

Fig. 7. Diffusivity of chlorpheniramine maleate in aqueous hydroxypropyl cellulose at $37^{\circ} \mathrm{C}$ (Data : Ref. 20).

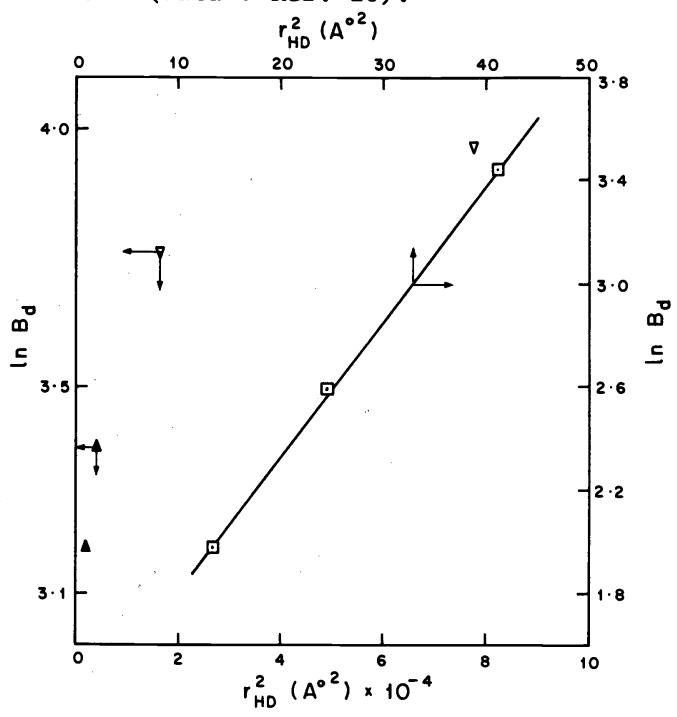

Fig. 9. Relationship of $\mathrm{B}_{d}$ and the molecular size of the diffusant for polyvinyl hydrogen phthalate $(\boldsymbol{\Lambda})$, polyanethol sulfonate $(\nabla)$ and glucose, maltose and maltotriose (回) (Data : Ref .25, 27).

determined by the parameter $B_{\text {d }}$ which is related to the critical free volume which must be created for the diffusant to accommodate itself into a hole. Following the arguments by Cohen and Turnbull (24), it is logical to deduce that a plot of in $B_{d}$ will be a linear function of the square of the diameter or the hydrodynamic radius $\left(r_{d}^{2}\right)$ of the diffusing molecule. Figure (9) shows a plot of $\ln B_{d} v s r_{d}^{2}$ based on the analysis of literature (25) data. The relationship could be seen to $d$ be approximately linear. Examination of the recent data presented by Nystrom and Roots (26) pertaining to diffusion of sucrose, $\beta-a l a n i n e$ and bovine serum albumin in hydroxypropyl cellulose solutions shows that for a given polymer concentration the relative diffusivity decreases with increasing molecular diameter of the diffusant.

Apart from the size, the shape of the diffusing molecule plays an important role in the diffusion process. Linear diffusants diffuse by a segmental mode as a result of which the effective hydrodynamic radius will have little or no relation to $B_{\text {. }}$. In order to verify this we analysed the diffusivity of linear polymers in sodium hyaluronate solutions (27). It was observed that there was no relation of the kind that we had observed earlier and that $B_{d}$ was in no way related to the effective hydrodynamic radius. It is interesting to see that similar conclusions have been drawn by Vrentas and Duda (28) regarding the dependence of the diffusivity on the size and shape of the diffusing molecule. 
Dependence of diffusivity on crystalline fraction. The models correlating diffusivity in semicrystalline polymers generally presume that the contribution of the crystalline phase to transport is negligible (Ref. 29-31). This is justifiable, since the close packing density in the crystalline phase renders the contribution of the crystalline region to the free volume of the system negligible (Ref. 32). Thus, from a free volume standpoint, the semicrystalline polymer can be considered as a two component system in which only one component is contributing to the free volume. The diffusion coefficient of a molecule in a semicrystalline polymer containing crystalline fraction $\emptyset_{c}$ can be deduced [in the same way as we deduced equations (6) and (7)]

$$
\ln \frac{D_{c}}{D_{a}}=-\frac{B_{d}}{f_{a}}\left[\frac{\emptyset_{c}}{1-\emptyset_{c}}\right]
$$

where $D_{\text {f }}$ is the diffusivity in the semicrystalline polymer, $D_{a}$ is the diffusivity in the fully afforphous polymer, $f$ is the fractional free volume in the amorphous polymer and $\emptyset_{c}$ is the volume fraction of a the crystalline portion. Figure 10 shows the plots based on equation (19) for diffusion of oxygen in polyethylene (data from Ref. 33) and on diffusion of CI disperse red 15 in polyethylene terephthalate (data from Ref. 34). As is evident, the trend predicted by equation (19) is borne out.

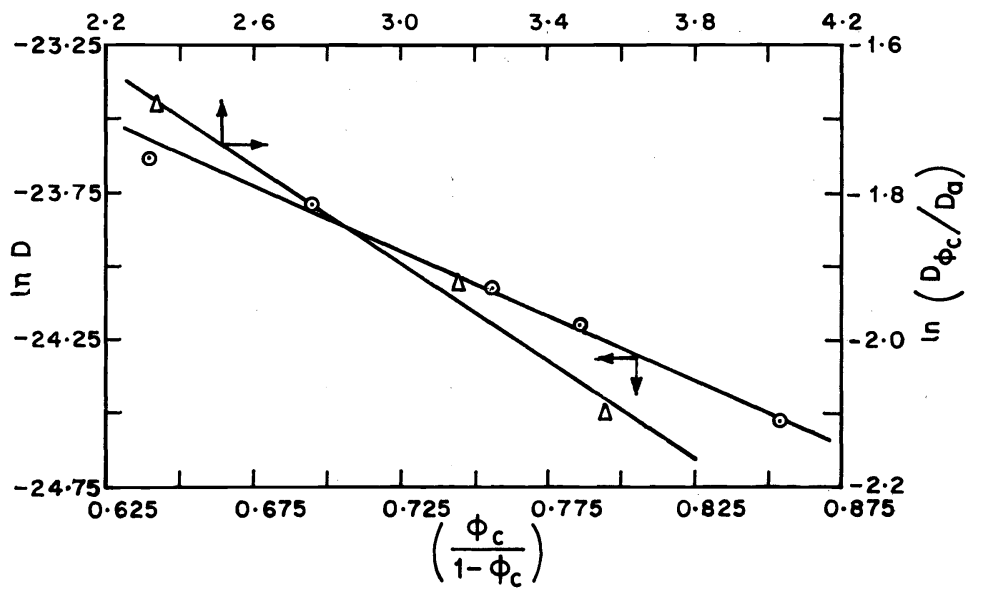

Fig. 10. Diffusivity of CI disperse red 15 in polyethylene terephthalate as a function of crystallinity $(\odot)$ (Data : Ref. 34) and oxygen in polyethylene ( $\Delta$ ) (Data : Ref. 33 ).

Influence of the diffusant size and shape. We have already discussed the influence of the size and shape of the diffusing molecule on $B_{d}$. Since $B_{d}$ is a measure of the minimum hole size required for the diffusing molecule to accommodate itself, it would be expected to increase with increasing size of the diffusing molecule. This fact has recently been substantiated by Vrentas and Duda (28) and by Kosiyanon and Wilcgregor (35).

Extending the arguments proposed earlier, we would expect $\ln B_{d}$ to be a linear function of the square of the reduced molecular diameter. Michaels and Bixler (36) studied diffusivities of a large number of diffusants in four hydrocarbon polymers. However, in this case both the crystali inity and the microstructure of the polymer was changed simultaneously. For comparative purposes, we assumed that for a given semicrystalline polymer containing crystalline fraction $\emptyset_{c}$, we have

$$
B_{d}=-\frac{\ln \left[\frac{{ }_{\emptyset_{c}}}{D_{0}}\right]}{\left[\frac{\emptyset_{c}}{1-\emptyset_{c}}\right]}
$$


Values of $B_{\text {f }}$ for each diffusant were calculated and plotted as a function of the molecular diameter. $d$ Figure 11 shows such a plot for high density polyethylene, thus confirming the trend predicted by us.

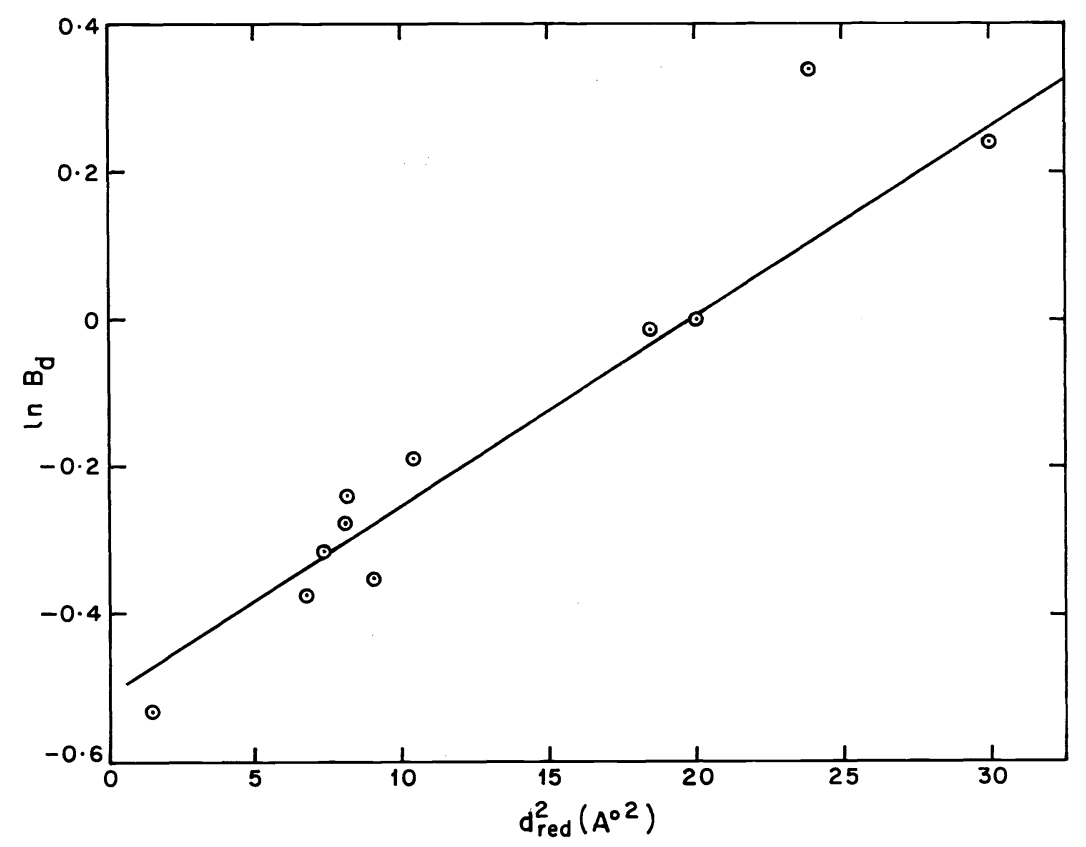

Fig. 11. Relationship between $B_{d}$ and the reduced molecular diameter of the diffusant (Date : Ref. 36).

The shape of the diffusing molecule too plays an important role in the diffusion process. Kline (37) investigated diffusion of linear molecules in semicrystalline polymers and showed that the structural attributes of an amorphous phase, which do not influence diffusivity of small molecules, play a key role in diffusion of long chain linear molecules. Consequently diffusivity of such molecules increases with increasing crystallinity. Such systems do not lie within the scope of the free volume model presented in this paper. For the same reason, the linear relationship of the kind observed in Fig. 11 is not expected to be valia for linear hydrocarbon penetrants such as normal paraffins.

\section{DIFFUSION IN CROSS-LINKED POLYMERIC SYSTEWIS}

Not many data are available on diffusion in cross-linked polymer systems. Barrer and coworkers $(38,39)$ found qualitatively that an increase in crosslinking leads to a decrease in diffusivity and the effect is more drastic, the larger the molecular size of the diffusing molecule. Kulkarni and Mashelkar (40) recently proposed a modified Maxwell relationship to correlate the diffusivities in crosslinked polymeric networks.

Influence of crosslining (chemical reaction)

Chemical crosslinking can be brought about by bridging the polymer chains e.g. vulcanization of elastomers or by building up polymeric networks starting from multifunctional monomers. Crosslinking leads to a decrease in molecular mobility within the matrix and therefore has a profound effect on mechanical, physical and electrical properties related to mobility of the polymer chains (Ref. 41).

Crosslinking leads to a decrease in the specific volume of the polymer (Ref. 42-44), which, in turn is related to the amount of crosslinking monomer by the relationship (41)

$$
\bar{v}=\bar{v}_{0}-\times \rho
$$

where $\bar{v}$ is the specific volume of the crosslinked polymer, $\bar{v}$ is the specific volume of uncrosslinked polymer $\rho$ is the number of moles of crosslinking monomer per gram of crosslinked polymer and $x$ is a constant of proportionality which is characteristic of the cross- 
linking monomer.

We assume that the occupied volume of the polymer is independent of the degree of crosslinking. The change in specific volume may then be assumed to reflect on the change in the free volume. Therefore, expressing the free volume in terms of the concentration of the crosslinking monomer, we can readily deduce the relationship.

$$
\frac{1}{\ln \frac{D}{D_{0}}}=-\frac{1}{B_{d}}\left[\frac{f_{0}^{2}}{x\left(\rho-\rho^{*}\right)}\right]+\frac{f_{0}}{B_{d}}
$$

where $D$ and $D_{0}$ denote the diffusivities of the diffusant in the crosslinked networks containing $\rho$ and $\rho^{*}$ moles of crosslinking monomer per gram of crosslinked polymer and $f_{0}$ denotes the free volume fraction in the network polymer containing $\rho^{*}$ moles of crosslinking monomer per gram of crosslinked polymer. As a specific case, if $\rho * i s$ zero ( $i$.e. if an uncrosslinked polymer is chosen as the reference state), equation (22) is reduced to

$$
\frac{1}{\ln \frac{D}{D_{0}}}=-\frac{1}{B_{d}}\left[\frac{f_{0}^{2}}{x \rho}\right]+\frac{f_{0}}{B_{d}}
$$

where $D_{0}$ and $f_{\text {now }}$ represent diffusivity and free volume fraction in the uncrosslinked polymer. Figures 12 and 13 confirm the results of these analyses of diffusivity data in crosslinked hydrogels.

Fig. 12 .

Diffusivity of progesterone in in EGDMA (०) and TEGDiMA $(\Delta)$ crosslinked HEMA (Lata : Ref. 45).

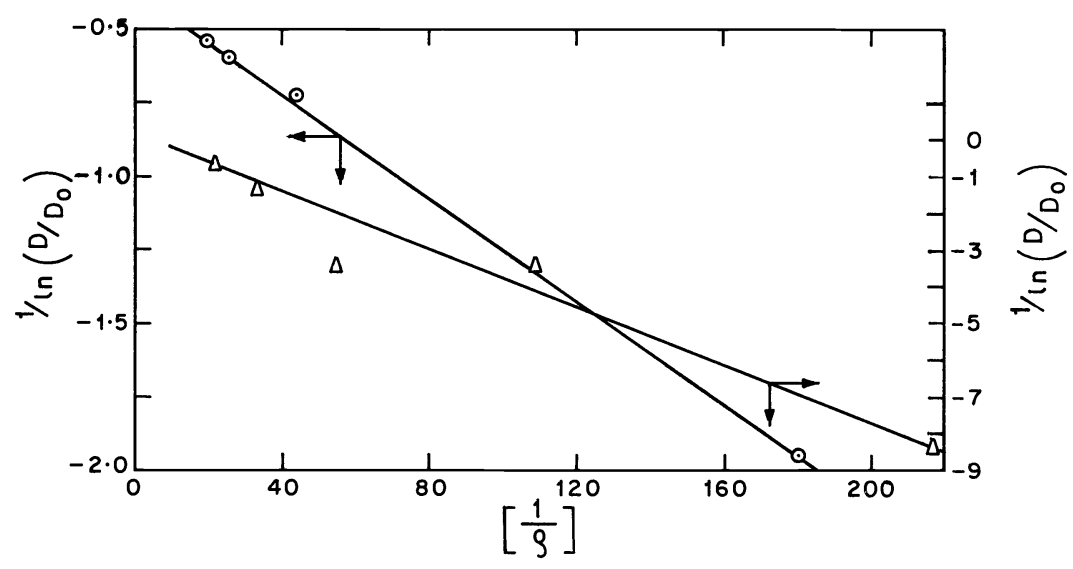

Fig. 13. Effect of crossl inking on diffusivity of norgestomet in hydrophilic implants (Data : Ref. 46).

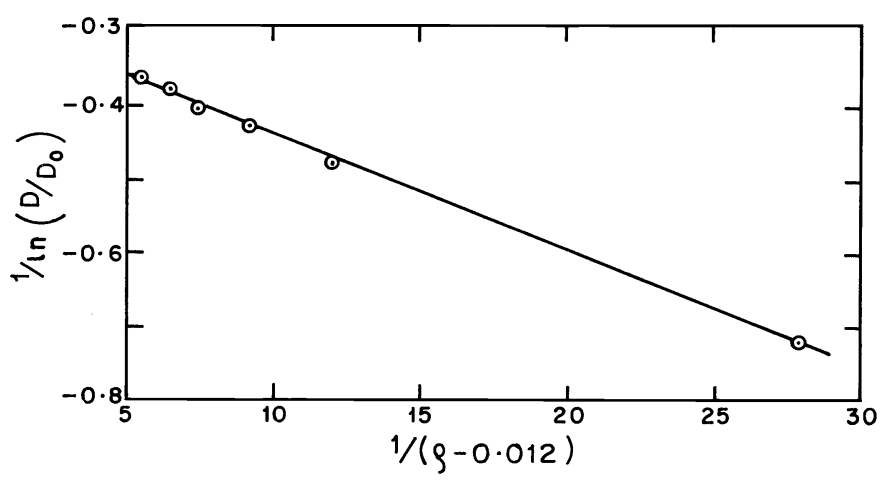


There have not been many systematic efforts to examine the effect of such variables as the nature of the crosslinking monomer, the size and shape of the diffusing molecule, temperature etc. However, same clear trends can be seen from the reported data. Figure 12 shows plats of relative diffusivity vs the amount of crosslinking monomer used to crosslink 2-hydroxyethyl methacrylate. It becomes immediately obvious that at a given level of crosslinking, the decrease in diffusivity is much more rapid in the case of ethylene glycoldimethacrylate crosslinked systems than in the case of tetraethylene glycoldimethacrylate crosslinked systems, which is to be expected.

Barrer et al. $(38,39)$ studied the influence of the extent of crosslinking on diffusivities of gases in sulphur crosslinked natural rubber and tetraethylene glycoldimethacrylate crosslinked acrylic polymers. In both the cases, the decrease in the diffusivity was more drastic, the greater the diameter of the diffusing molecule.

Influence of crosslinking (irradiation)

Irradiation can lead to both crosslinking and chain scission. Crosslinking would lead to a decrease in free volume whereas chain scission would lead to an increase in free volume. Therefore, depending upon the relative importance of the two types of processes, irradiation would lead to either a decrease or an increase in the diffusion coefficient. Wilson (47) has summarised the effects of ionizing radiations on polymers. Polymers such as polyethylene, polypropylene, polystyrene etc. undergo mainly crosslinking whereas acrylics, fluorinated polymers etc. undergo chain scission (47). One would, therefore, imagine that the framework developed in the foregoing will hold for the former class of polymers.

In order to quantify the results in terms of the radiation dosage, we make use of the parameter $\mathbf{G}$. which signifies the number of crosslinks formed in one gram of polymer per $100 \mathrm{eV}$ of radiation absorbed (48). A value of $\mathrm{G}_{\mathrm{cl}}=3$ reported for polye thylene in the literature is equivalent to $8.716 \times 10^{-3}$ mole $\mathrm{cl}$ percent crosslinks per $\mathbf{M}$ rad dose. We have already shown that there exists a linear relationship between $\left[\mathrm{ln}\left(\mathrm{D} / \mathrm{D}_{0}\right)\right]^{-1}$ and where $\rho^{-1}$ represents the mole of crosslinking monomer per gram of polymer. Consequently, a plot of $\left[\ln \left(\mathrm{U} / \mathrm{D}_{0}\right)\right]^{-1}$ vs $1 /[\mathrm{M} \mathrm{rad}]^{-1}$ should be linear. In Fig. 14 we show the influence of irradiation in vacuum as well as in air on diffusivity of methane and nitrogen in polyethylene. It is seen that the trend predicted by equation (22) is borne out.

Fig. 14.

Influence of radiation crosslinking on diffusivity of methane $(\Delta)$ and nitrogen $(\boldsymbol{v})$ in polyethylene (Data : Ref.49).

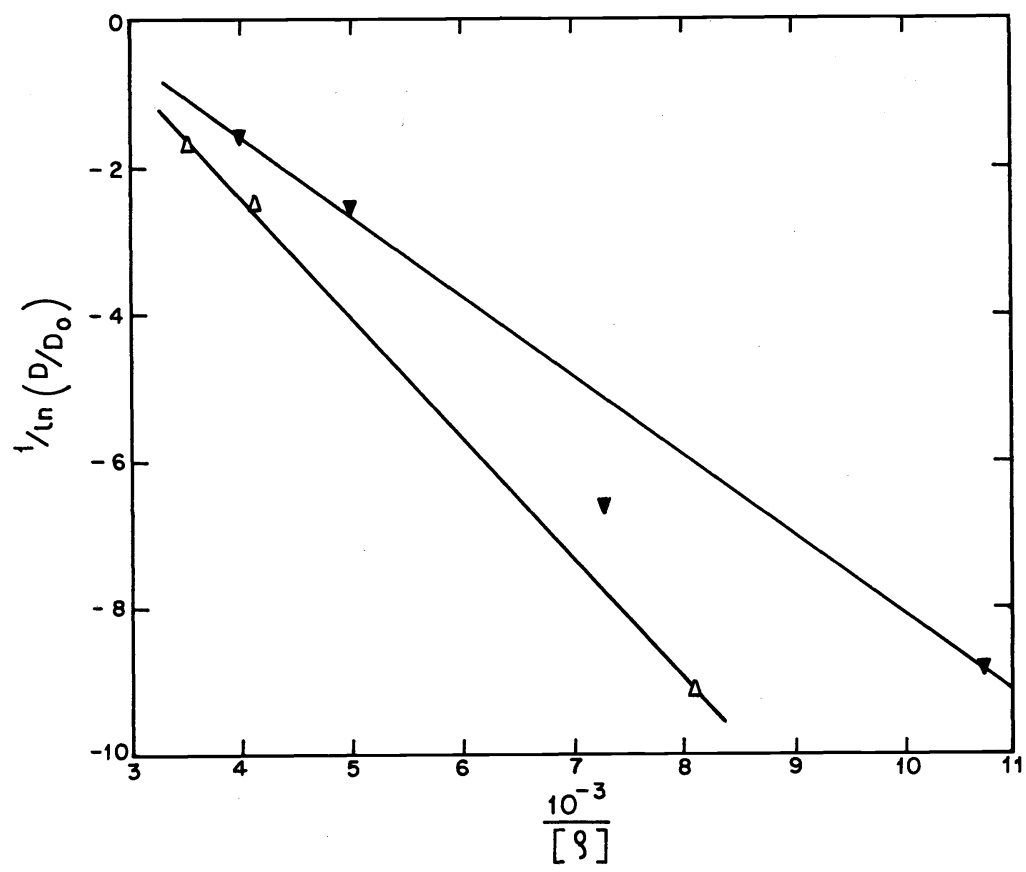

Diffusion in filled polymers

The incorporation of the fillers into polymers can affect the diffusional transport in three ways. (1) From a free volume view point, the filler particles do not contribute to free volume, thus giving rise to obstruction effect as in the case of semicrystalline polymers. (2) The polymer chains get adsorbed on the filler particles and therefore the molecular mobility within the segments in the neighbourhood of the particle gets restricted. The 
effect is somewhat analogous to the immobilization of polymer chains in the amorphous regions resulting from the fact that a part of the chain in the amorphous region is also present in the crystalline region which restricts the mobility of the polymer chain as a whole. (3) In case the filler particle is not completely wetted by the polymer, the diffusing molecule can also get adsorbed on the filler particle. The effect of the adsorption of the diffusing molecule itself will not be reflected in the case of diffusivity measurements based on steady state methods.

The obstruction effect of the filler particle can be taken into consideration by assuming its contribution to free volume to be zero. The immobilization of polymer chains due to adsorption on the filler particle will result in a decrease in the mobility of polymer chains in the vicinity of the particle, thereby causing a decrease in the diffusivity. We therefore, propose that the decrease in diffusivity resulting from immobilized chains of this kind can be viewed as though the chains were to have the same extent of mobility and were to be densely packed in such a way that their contribution to free volume was lower.

We now assume that the number of chains of the polymer so immobilized and hence the resulting equivalent decrease in the free volume of the system is proportional to the volume fraction of the filler in the system. We can then write

$$
f_{f}=f_{0}\left(1-\emptyset_{F}\right)-k \emptyset_{F}
$$

where $f_{f}$ is the fractional free volume of the filled polymer, fo that of the unfilled polymer, $\emptyset_{F}$ is the volume fraction of the filler and $K$ is an immobilization parameter.

Using the AFVS model as in the earlier cases, we can deduce

$$
\frac{1}{\ln \frac{D_{f}}{D_{0}}}=-\frac{f_{0}}{B_{d}}\left[\frac{f_{0}}{f_{0}+k}\right] \frac{1}{\emptyset_{F}}+\frac{f_{0}}{B_{d}}
$$

Thus, if the above model were to be valid, a plot of $\left[\ln \left(U_{f} / D_{0}\right)\right]^{-1}$ vs $\left(\emptyset_{F}\right)^{-1}$ should be linear. Analysis of the literature data on diffusion data on diffusion in $f_{i l l e d}$ polymer within the framework of equation (25) confirms the validity of our approach (Figs. 15, 16). Analysis of the data further reveals that at a given filler loading for a filler such as carbon black diffusivity of a molecule such as benzanthracene falls more rapidly than that of hexadecane which can diffuse by segmental motion.

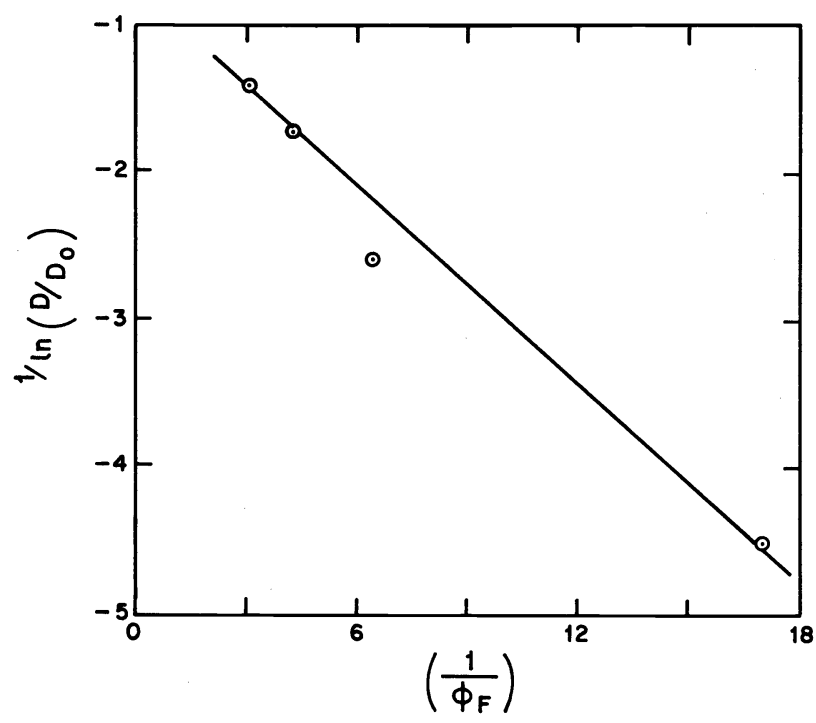

Fig. 15. Influence of carbon black loading on diffusivity of benzanthracene in SBR (Data : Ref. 50). 
Fig. 16. Influence of glass microsphere loading on diffusivity of nhexadecane in cispolybutadiene (Uata : Ref.51).

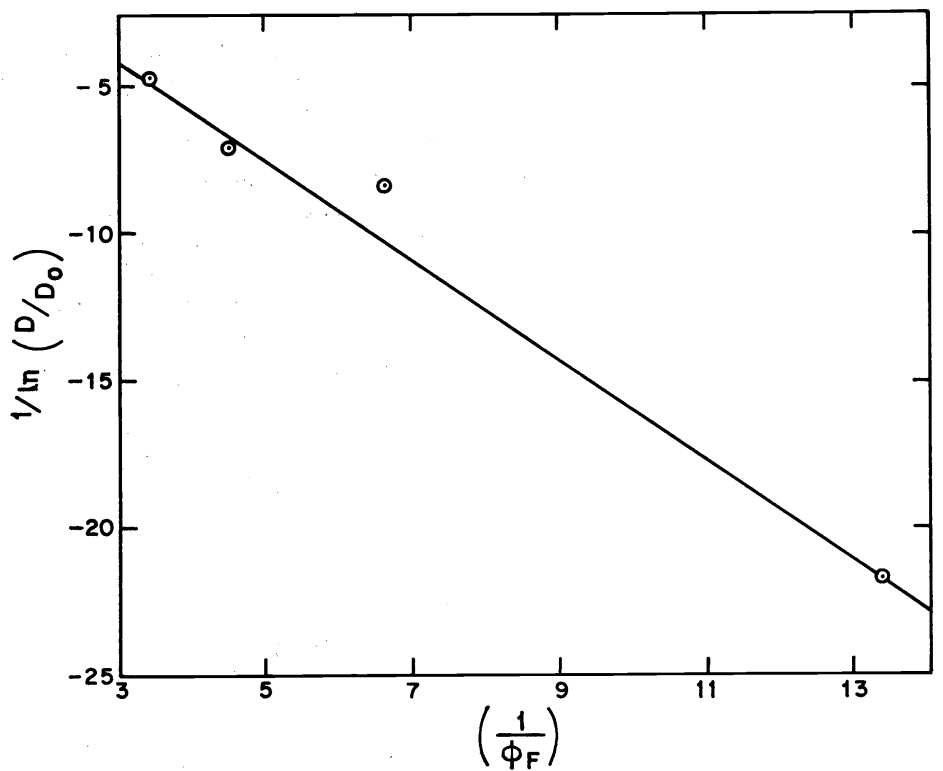

Diffusion in polymer blends

The polymer blends can be considered to consist of a continuous phase in which a second polymeric phase is dispersed. Transport properties of such two phase systems can be correlated within the framework of viaxwell equation (Ref. 53).

Blending can be considered to lead to an alteration in the free volume state of the parent polymer because of the presence of the added polymer. The free volume state of a polymer blend having a volume fraction of $\emptyset_{\mathrm{BL}}$ of an added polymer can be approximated by using the linear additivity law. It is then obvious that equation (18) should al so correlate composition dependence of diffusivities in polymer blends. Plots of relative diffusivity vs blend composition (Fig. 17) confirm the utility of free volume approach in correlating diffusivity data in polymer blends.

Fig.17. Uiffusivity of nitrogen (॰) and oxygen $(\Delta)$ in PVCINBR blends as a function and blend composition (Lata : Ref.54).

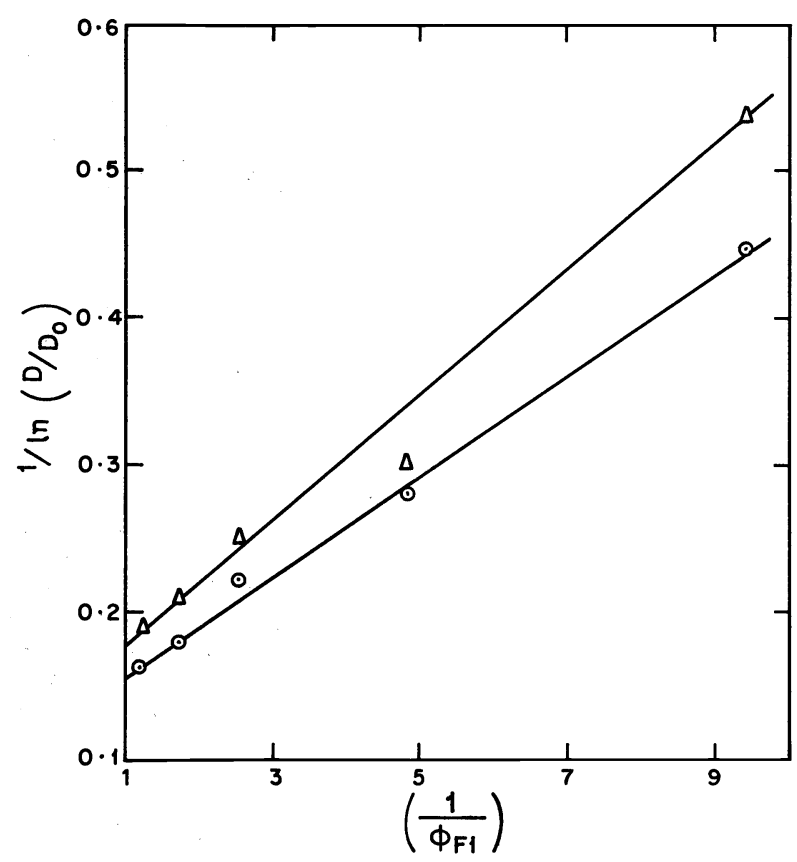


Diffusion in drawn fibres

By relating the free volume with the material properties, it is possible to take into account the influence of parameters which govern the transport properties directly or indirectly. For instance, during the drawing of polyamide fibre of a lower degree of crystallinity, orientation induced crystallization sets in, which leads to an increase in the density of the fibre (55). On the contrary, drawing of a highly crystalline fibre leads to a rupture of crystallites and hence to a decrease in density. Hattori (55) has shown that there exists a linear relationship between the specific gravity of the fibre and the draw ratio. We are, therefore, justified in assuming that the specific volume and hence the free volume will vary approximately linearly with the draw ratio. Choosing the maximum draw ratjo $r^{*}$ as the reference state, we can consider that the fibres at all draw ratios less than $r^{*}$ are at a higher free volume state, and hence diffusivity of a dye in such fibres will be higher. Using $r$ for $f$ in equation (23) and reorganizing, we get

$$
\frac{1}{\ln \frac{D}{D_{0}}}=-\frac{f\left(r^{*}\right)}{B_{d}}\left[\frac{f\left(r^{*}\right)}{K\left(r^{*}-r\right)}-1\right]
$$

Where $U$ and $U$ correspond to the diffusivities in fjbres drawn to the draw ratios $r$ and $r^{*}$, respectively. Thus, a plot of $\left[\ln \left(D / D_{0}\right)\right]^{-1}$ vs $\left[r^{*}-r\right]^{-1}$ should be linear. Figure 18 shows a plot for diffusivity of xylene fast blue $2 P$ in nylon 6 in the axial direction as a function of draw ratio. A draw ratio of 5.2 has been chosen as a reference. The observed linearity is again in accordance with our predictions.

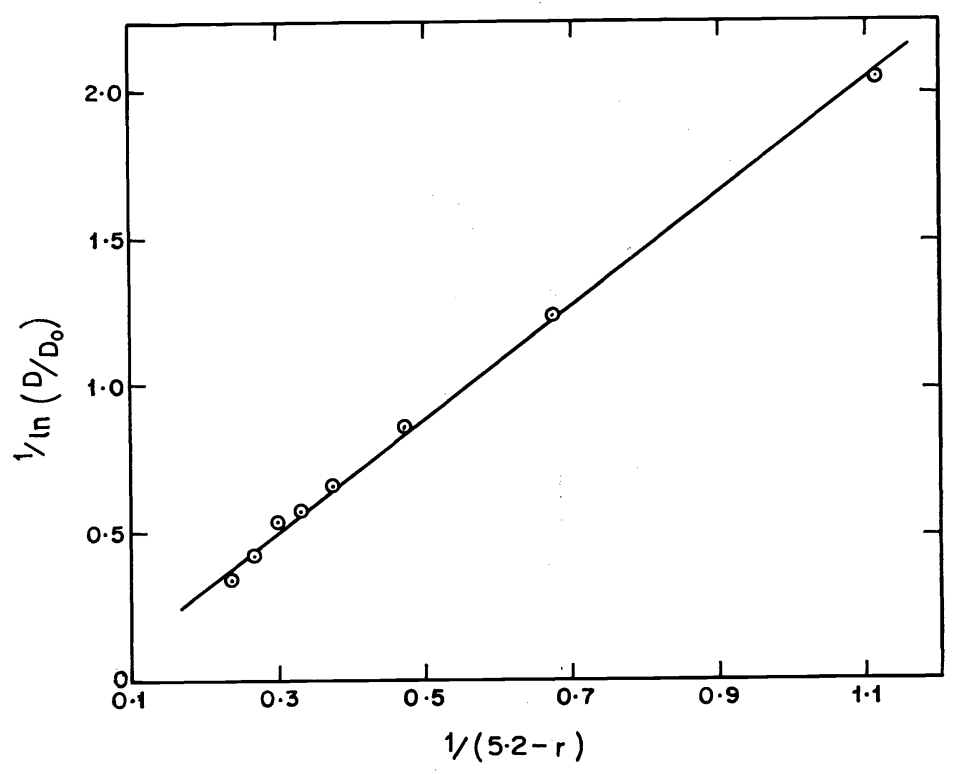

Fig. 18. Influence of draw ratio on diffusivity of xylene fast blue $2 \mathrm{P}$ in nylon 6 at $80^{\circ} \mathrm{C}$ (Data : Ref. 56).

\section{THERMAL CONDUCTION IN POLYMERS}

The mechanism of thermal conduction in polymers is not as clearly uncierstood as that of viscous or diffusive transport in polymers. Several approaches have been putforth in the literature (Ref. 57, 58). Sheldon and Lane (59) considered that the thermal conduction in polymers is a result of molecule to molecule transfer of energy by translational, rotational or vibrational modes and that these processes are diffusional in nature. It is therefore likely that the thermal conduction in polymers could be a free volume controlled process. The fact that the temperature dependence of thermal conduction in polymers obeys WLF relationship (Ref. 60) in some cases lends further support to this hypothesis.

Thermal conduction in polymer solutions As discussed in the prior section on diffusion, the addition of a polymer to a solvent decreases the free volume of the system. Now whereas a decrease in the free volume leads to a decrease in the diffusivity, the themal conductivity increases. This could be explained 
on the basis of the fact that the monomers constituting the polymer are linked up with one another by primary valence bonds, whereas the solvent molecules exert only secondary valence forces, and as is well known (Ref. 58) the secondary valence forces offer a higher resistance to thermal conduction than the primary valence forces. It is therefore obvious that the thermal conductivity of the solution should increase with increasing polymer concentration. In general we can assume that a reduced free volume will lead to an increase in thermal conductivity. Extending arguments similar to those used in the development of earlier transport equations, we get,

$$
\lambda=A_{\lambda} \exp \frac{B_{\lambda}}{f}
$$

where $\lambda$ is the thermal conductivity of the medium under consideration, $A_{\lambda}$ is the preexponential factor and $B_{\lambda}$ is the jump factor. Following arguments similar to those used in the development of equation (7) we can deduce an expression in the case of a polymer solution

$$
\frac{1}{\ln \frac{\lambda}{\lambda_{0}}}=\frac{f(0)^{2}}{{ }^{B} \delta} \quad \frac{1}{\theta_{p}}+\frac{f(0)}{B_{\lambda}}
$$

Here $\lambda$ and $\lambda_{0}$ denote the thermal conductivity of the polymer solution and the pure solvent, respectively. $f(0)$ denotes the fractional free volume of the pure solvent, $\delta$ denotes the difference in the fractional free volume of the polymer and the solvent and $\rho_{p}$ denotes the polymer concentration. Figure 19 shows the verification of equation (28) for thermal conduction in polystyrene solutions in toluene.

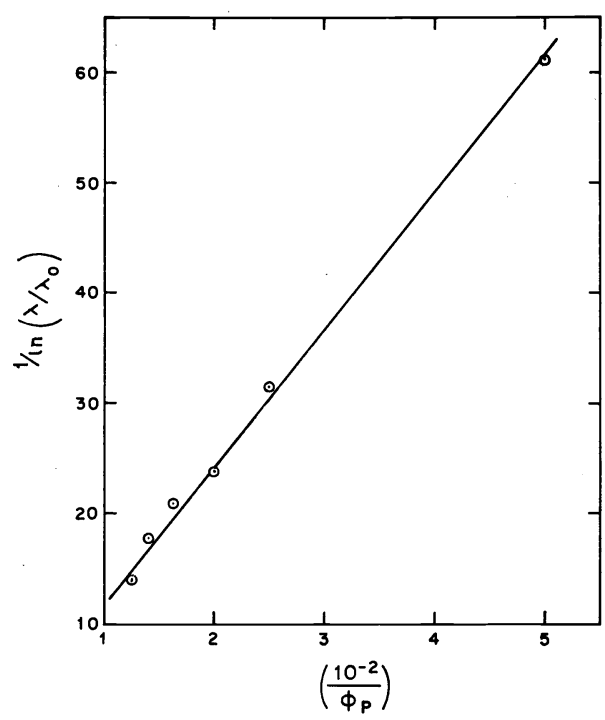

Fig. 19. Thermal conductivity of polystyrene-toluene solutions (Data: Ref. 61).

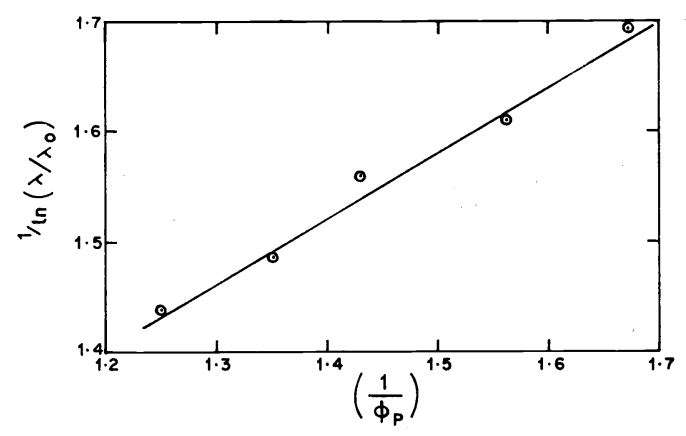

Fig. 20. Thermal conductivity of PVCdialphanyl phthalate system as a function of polymer concentration (Data : Ref. 59).

Thermal conduction in plasticized polymers

The above arguments can be used while analysing the data on thermal conduction in plasticized polymers, provided $\delta$ is interpreted as the difference in fractional free volumes of the pure polymer and the plasticizer. Equation (28) can then be used for analysing the data. Figure (20) shows the verification of equation (28) for thermal conduction data for plasticized polyvinyl chloride.

Thermal conduction in semicrystalline polymers

The crystalline domains present in a semicrystall ine polymer possesses higher degree of molecular order in comparison to the amorphous polymers. Therefore such polymers also 
possess a higher thermal conductivity. Interpreting the role of fractional free volume in the same way as we have done while deducing diffusivities in semicrystalline polymers we can readily arrive at the following equation to correlate the thermal conduction data in semicrystalline polymers.

$$
\frac{1}{\ln \frac{\lambda_{c}}{\lambda_{a}}}=\frac{f(a)^{2}}{{ }^{B_{\lambda} \xi}} \frac{1}{\theta_{c}}+\frac{f(a)}{B_{\lambda}}
$$

where $\lambda_{c}$ and $\lambda_{a}$ are thermal conductivities in the semicrystalline polymer, and a purely amorphous polymer, $f_{a}$ is the fractional free volume of the parent amorphous matrix, $\xi$ is the difference between the fractional free volume of purely amorphous and crystalline regiones, and $\emptyset_{C}$ is the crystalline volume fraction. Figure 21 provides verification of this equation for correlating thermal conduction in polyethylene.

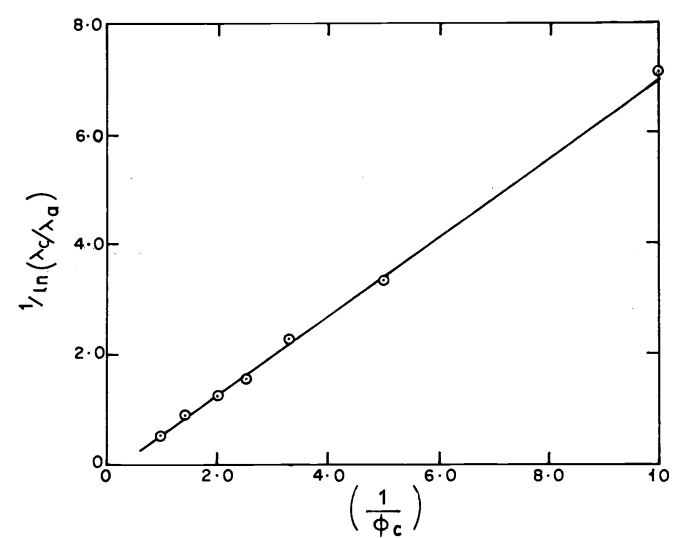

Fig. 21. Thermal conductivity of polyethylene as a function of crystallinity (Data : Ref. 62).

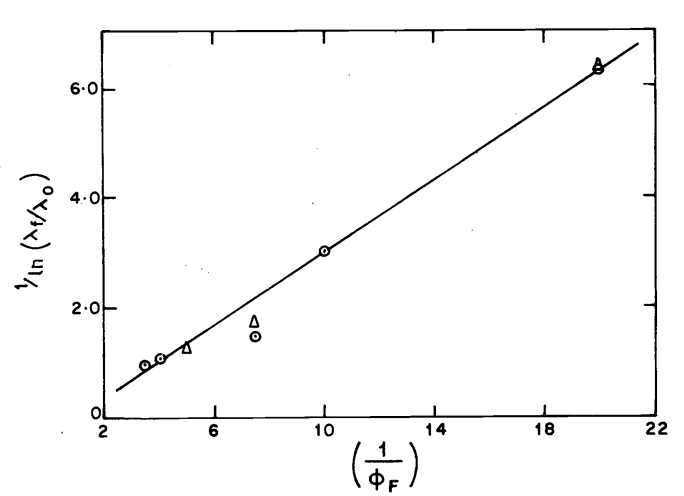

Fig. 22. Thermal conductivity of polystyrene as a function of $\mathrm{CaO}(\mathcal{O})$ and Mg0 $(\Delta)$ volume fraction (Data : Ref. 63).

Thermal conduction in filled polymers

A conducting filler material acts in the same way as is implicit in the two phase model of a semicrystalline polymer, where the role of the crystalline fraction is assumed to be the same that of the filler particle. We are again able to deduce an expression which relates the thermal conductivity in filled polymer $\left(\lambda_{f}\right)$ to that in the unfilled polymer $\left(\lambda_{0}\right)$

$$
\frac{1}{\ln \frac{\lambda_{f}}{\lambda_{0}}}=\frac{f(0)^{2}}{{ }^{B} \lambda_{\lambda}} \quad \frac{1}{\theta_{F}}+\frac{f(0)}{{ }^{B}{ }_{\lambda}}
$$

Here $f(0)$ is the fractional free volume of the pure polymer matrix, $\delta$ is the difference between the free volume fraction of the unfilled polymer and the filler and $\emptyset_{F}$ is the volume fraction of the filler particles. Figure 22 shows the verification of equation (30) for thermal conductivity of polystyrene calcium oxide and polystyrene magnesium oxide filled systems. It is interesting to note that the data for both calcium oxide and magnesium oxide fillers superimpose.

Concluding remarks

We have demonstrated in the foregoing the success of AFVS model in correlating and analysing a diverse variety of transport processes in diverse polymeric media. In each case, only limited data have been presented, but a far greater elaboration and extensive testing of data has been provided by Kulkarni and Mashelker $(21,64)$.It is decidedly true that the model will fail in many specific circumstances, since such a unique framework cannot be expected to handle all the possible diversities of existing phenomena. However, the development presented in the foregoing is important from two view points. In the first instance, it does show the possibility of evolving a unified free volume based framework and secondly, in the absence of data, it provides a useful engineering tool for estimation purposes. Additionally, it also provides directions for future research, which might elaborate some of the simple model concepts presented here. 


\section{REFERENCES}

1. M. Kurata and W.H. Stockmeyer, Adv. Polym. Sci. 3, 196-312 (1961).

2. G.C. Berry and T.G. Fox, Adv. Polym. Sci. 5, 261-357 (1968).

3. V. Semjonow, Adv. Polym. Sci. 5, 387-450 (1968).

4. H.C. Frisch and R. Simha, Rheology, 1, 525-613 (1956).

5. S.H. Maron and I.H. Krieger, Rheology, 3, 121-143 (1960).

6. M.L. Williams, R.F. Landel and J.D. Ferry, J. Am. Chem. Soc. 77, $3701-3707$ (1955).

7. H. Fujita, Adv. Polym. Sci. 3, 1-47 (1961).

8. B. Chitrangad and H.R. Osmers, J. Polym. Sci. (Phys. Edn.) 18, 1219-1236 (1980).

9. J.V. Robinson, Trans. Soc. Rheol. 1, 15-24 (1957).

10. T. Kataoka, T. Kitano, Y. Oyanagi and M. Sasahera, Kheol. Acta 18, 635-647 (1979).

11. H. Van Oene, Polymer Blends, p. 295, Academic Press, New York (1978).

12. K. Ninomiya and J.D. Ferry, J. Colloid. Sci. 18, 421-432 (1963).

13. H. Leaderman, R.G. Smith and L.C. Williams, J. Polym. Sci. 36, 233-257 (1959).

14. C.D. Han, Rheology in Polymer Processing, p. 165, Academic Press, New York (1976).

15. A. Rudin and G.B. Strathdee, J. Paint Technol. 46, 33-43 (1974).

16. L.U. Utracki, R. Simha and iN. Eliezer, Polymer, 10, 43-54 (1969).

17. R.W. Fillers and N.W. Tschoeg1, Trans. Soc. Rheo T. 21, 51-58 (1977).

18. G. Astarita and R.A. Nashelkar, The Chem. Engr. 317, 100-105 (1977).

19. S.U. Li and J.L. Gainer, I and EC (Fundam.), $\underline{7}, \overline{433}-440$ (1968).

20. H. Lapidus and N.G. Lordi, J. Pharm. Sci., 57, 1292-1301 (1968).

21. M.G. Kulkarni and K.A. Mashelkar, Chem. Engg. Sci. (in press).

22. V.A. Bagdonaite, S.A. Juskeviciute and Yu. A. Sh1yapnikov, Poiymer, 22, 145-146 (1981).

23. K. Namikawa, H. Okazaki, iN. Nakanishi, R. ivatsuno and T. Kamikubo, Agri. Biol. Chem. 41, 1003-1009 (1977).

24. M.H. Cohen and D. Turnbul1, J. Chem. Phys. 31, 1164-1169 (1959).

25. K. Nakanishi, S. Adachi, S. Yamamato, R. Matsuno, A. Tanaka and T. Kamikubo, Agri. Biol. Chem. 41, 2455-2462 (1977).

26. B. Nystrom and J. Roots, Eur. Polym. J. 16, 201-205 (1981).

27. J.C. Laurent, B.N. Preston, H. Pertoft, $\bar{B}$. Gustaffson and M. MicCabe, Eur. J. Biochem. 53, 129-136 (1975).

28. J.S. Vrentas and J.L. Duda, J. Polym. Sci. (Phys.Edn.) 17, 1085-1096 (1979).

29. H.A. Bent, J. Polym. Sci. 24, 387-400 (1957).

30. S.W. Lasoki and W.H. Cobbs, J. Polym. Sci. 36, 21-33 (1959).

31. C.H. Klute, J. App1. Polym. Sci. 1, 340-350 (1959).

32. A. Peterlin, Makromol. Chem. Supp. 3, 215-232 (1979).

33. A.S. Michaels and R.B.Parker, J. Polym. Sci. 41, 53-71 (1959).

34. J.H. Dumbleton, J.P. Bel1 and T. Murayama, J. App1. Polym. Sci. 12, $2491-2505$ (1968).

35. R. Kosiyanon and R.M. Mcgregor, J. Appl. Polym. Sci. 26, 629-641 (1981).

36. A.S. Michaels and H.J. Bixler, J. Polym. Sci. 50, $413-439$ (1961).

37. J. Kline, J. Polym. Sci. (Phys. Edn.) 15, 2057-2064 (1977).

38. R.M. Barrer, Trans. Far. Soc. 35, 628-656 (1939).

39. R.M. Barrer and G. Skirrow, J. Polym. Sci. 3, 549-563 (1948).

40. M.G. Kulkarni and R.A. Mashe 1kar, Polymer, 22, 1658-1664 (1981).

41. L.E. Nielsen, Reviews in Macromolecular Chemistry, 4, 69-104 (1970).

42. T.G. Fox and S. Loshak, J. Polym. Sci. 25, 371-390 T1955).

43. P. Mason, Polymer, 5, 625-635 (1964).

44. K. Shibagama and Y. Suzuki, J. Polym. Sci. A3, 2637-2651 (1965).

45. G.M. Zentner, J.R. Cardinal and S.W. Kim, J.Pharm. Sci. 67, 1352-1355 (1978).

46. Y.W. Chien and E.P.K. Lau, J. Pharm. Sci. 65, 488-492 (1976).

47. J.E. Wilson, Radiation Chem istry of Fionomers, Polymers and Plastics,

p. 373 Marcel1 Dekker, New York (1972).

48. A.R. Schultz, Encyclopedia of Polymer Science and Technology, 4,331-414 (1966).

49. P.J.F. Kanitz and R.Y.M. Huang, J. App1. Polym. Sci. 14, 2739-2751 (1970).

50. B.G. Coman, M.L. Deviney and L.E. Whittington, Rubber Chem. Technol. $45,278-308$ (1972).

51. C.K. Rhee and J.D. Ferry, J. App 1. Polym. Sci., 21, 467-476 (1977).

52. Y.J. Shur and B. Ranby, J. Appl. Polym. Sci., 19 , 2143-2155 (1975).

53. T.M. Connelly and J.C.R. Turner, Chem. Eng. Sci. 34, 319-324 (1979).

54. Y.J. Shur and B. Ranby, J. Appl. Polym. Sci. 19, 2143-2155 (1975).

55. H. Hattori and Y. Takagi, Bu11. Chem. Soc. Japan, 36, 675-681 (1963).

56. Y. Takaji, J. Appl. Polym. Sci. 9, 3887-3896 (1951).

57. K. Uberreiter and S. Nens, Kol10id Z., 123, 92-99 (1951).

58. K. Eierman, Rubber Chem. Technol. 39, $841-857$ (1966).

59. R.P. Sheldon and K. Lane, Polymer $\frac{6}{6}, 77-83$ (1965).

60. M.G. Kulkarni and R.A. Mashelkar, Polymer 22, 867-869 (1981).

61. R.J. Brunson, J. Chem. Engg. Data 20, 435-436 (1975).

62. K. Eiermann, Kunststoffe, 55, 335-338 (1965).

63. D.W. Sundstrom and Y.D. Lee, J. Appl. Polym. Sci. 16, 3159-3167 (1972).

64. |vi.G. Kulkarni and R.A. Mashelkar, in preparation 